\title{
Noisy Nonlinear Gene Regulatory Networks Analysis Using Ensemble Kalman Filter Based Particle Filter without a Model
}

\author{
Haixin Wang \\ Department of Mathematics and Computer \\ Science \\ Fort Valley State University \\ 1005 State University Dr \\ Fort Valley, GA 31030 \\ Phone number:14788273149 \\ wangh@fvsu.edu
}

\author{
Dawit Aberra \\ Department of Mathematics and Computer \\ Science \\ Fort Valley State University \\ 1005 State University Dr \\ Fort Valley, GA 31030 \\ Phone number: 14788256430 \\ aberrad@fvsu.edu
}

\begin{abstract}
In this paper, we propose a novel ensemble Kalman filter based particle filter for gene regulatory networks (GRNs) analysis, which incorporates ensemble Kalman filter into particle filter. New particles generated by particle filter are sampled by ensemble Kalman filter, which can take current measurements into account to predict the system states. This will alleviate the sample degeneracy problem in particle filter. The proposed method is model-free algorithm. Both particle filter and ensemble Kalman filter can be applied when the model is unknown, noisy, and nonlinear. This combination of approaches results in comparable accuracy, efficiency, and robustness. In the GRNs analysis, simulation results show that the proposed ensemble Kalman filter based particle filter performs better than particle filter in identifying dynamics relations among genes.
\end{abstract}

\section{CCS Concepts}

-Applied computing $\rightarrow$ Systems biology;

\section{Keywords}

gene regulatory networks;noise analysis;ensemble Kalman filter;particle filter

\section{INTRODUCTION}

The analysis of GRNs is one necessary step to discover drugs for gene related disease. With more time series data available, it becomes possible to find GRNs. GRNs are inherently noisy and nonlinear. Generally, GRNs can be par-

Permission to make digital or hard copies of all or part of this work for personal or classroom use is granted without fee provided that copies are not made or distributed for profit or commercial advantage and that copies bear this notice and the full citation on the first page. Copyrights for components of this work owned by others than ACM must be honored. Abstracting with credit is permitted. To copy otherwise, or republish, to post on servers or to redistribute to lists, requires prior specific permission and/or a fee. Request permissions from permissions@acm.org.

CSBio '16, December 19-22, 2016, Macau, Macao

(c) 2016 ACM. ISBN 978-1-4503-4794-5/16/12 . \$ $\$ 15.00$

DOI: http://dx.doi.org/10.1145/3029375.3029378 titioned into two categories: linear models and nonlinear models.

\subsection{Linear GRNs}

The regulators in linear GRNs contribute to the inputs of regulate functions in additive manner [1]. The linear models are efficient to infer large GRNs with high level abstraction. In [1] and [4], the authors demonstrated the linear model in numero and real expression data sets. Another benefit for linear model is to process noise using many available linear filters. A system of ordinary differential equations(ODEs) is transformed into a linear additive regulation model with noise. The expression level of a gene at a certain point can be calculated by the weighted sum of the expression levels of all genes in the network at a previous time point [3]. It may be represented by ODEs

$$
\frac{d e_{i}}{d t}=\sum_{j=1}^{N} w_{i j} e_{j}+\sum_{k=1}^{K} \nu_{i k} u_{k}+\beta_{i}
$$

where $e_{i}$ is the gene expression level of the $i^{t h}$ gene. $N$ is the number of genes in the GRNs. $w_{i j}$ represents the effect of the $j^{t h}$ gene on the $i^{t h}$ gene. Negative $w_{i j}$ means inhibition while positive $w_{i j}$ represents activation. $u_{k}$ is the $k^{t h}$ external (control) variable. $\nu_{i k}$ represents the effect of the $k^{t h}$ external variable on the $i^{t h}$ gene. $K$ is the number of external variable. $\beta$ is a bias term. In [2], the noise in the input data is considered in the linear additive regulation model. For each new input data, there is small amount of Gaussian noise with the same standard deviation added. In those models, steady states are obtained by solving linear programming problems.

\subsection{Nonlinear GRNs}

Compared with linear models, nonlinear models are computationally intensive and require more data. However, nonlinear models can reveal complex dynamic relations among genes in more details, which approach reality.

Nonlinear models involving ODEs are examples of nonlinear continuous models. Assuming there are $N$ genes of interest and $x_{i}$ denotes the state (such as the microarray 
reading) of the $i^{t h}$ gene, then the dynamics of the GRN may be modeled as

$$
\frac{d x_{i}}{d t}=f_{i}\left(x_{1}, x_{2}, \cdots, x_{N}\right)+\nu_{i} \quad i=1,2, \cdots, N .
$$

where $\nu_{i}$ is external noise.

Functions $\left(f_{i}, \forall i\right)$ are nonlinear functions to be estimated.

If each $f_{i}$ is a polynomial, the model is a polynomial model [9]. In [9], genetic programming is applied to infer the terms of polynomial equations. The constants are estimated by linear filters. The complexity is $\Omega\left(N^{2}\right)$. Therefore, it is hard to apply this model to higher dimensional system.

In [12], sigmoidal function is applied. The functions $\left(f_{i}, \forall i\right)$ are in the form

$$
\begin{array}{r}
\frac{d x_{i}}{d t}=\frac{C_{i, 1}}{1+e^{-\left\{\sum_{j=1}^{n}\left[\left(w_{i j}+\mu_{i j}\right) x_{j}\right]+\beta_{i}\right\}+\nu_{i}}}-C_{i, 2} x_{i} \\
i=1,2, \cdots, N .
\end{array}
$$

where $C_{i, 1}$ and $C_{i, 2}>0$ are two parameters. $\beta_{i}$ is an input and $w_{i j}$ is the weight value for input $j$ on gene $i . \mu_{i j}$ and $\nu_{i}$ are intrinsic noise and external noise, respectively. However, this model is limited. It can be applied to certain GRNs only.

S-system is a promising model to capture the rich dynamics of GRNs [7, 8, 13]. The S-system model is given by:

$$
\frac{d x_{i}}{d t}=\alpha_{i} \prod_{j=1}^{N} x_{j}^{g_{i, j}}-\beta_{i} \prod_{j=1}^{N} x_{j}^{h_{i, j}},(i=1, \ldots, N)
$$

where $x_{i}$ are state variables. $\alpha_{i}$ and $\beta_{i}$ are positive rate constants. $g_{i, j}$ and $h_{i, j}$ are exponential parameters called kinetic orders. If $g_{i, j}>0$, gene $j$ will induce the expression of gene $i$. On the contrary, gene $j$ will inhibit the expression of gene $i$ if $g_{i, j}<0 . \quad h_{i, j}$ will have the opposite effects on controlling gene expressions compared with $g_{i, j}$. The S-system is a quantitative model, which is characterized by power laws of exponential functions. It has a rich structure capability of capturing various dynamics in many biochemical systems. In addition, the S-system model has been proven to be successful in modeling GRNs $[13,7,8]$. However, the complexity is intensive. The structure is fixed. The time complexity of exponent estimation is high. These limit the application of S-system in GRNs.

In this paper, GRNs are analyzed without parametric prediction using ensemble filter based particle filter. The contribution of paper is to overcome the overfitting problem introduced by deterministic and stochastic models. If the model overfits data, it will sacrifice the predictive accuracy. In the proposed method, there is no model involved. It is not necessary to balance between the minimization of error and actual GRNs like deterministic and stochastic models. The paper includes four sections. In section two, the particle filter and ensemble Kalman filter are formulated, and the proposed ensemble Kalman filter based particle filter algorithm is given in detail. In section three, models are simulated using synthetic and real microarray data with discussions. Section four is for the conclusion of the research.

\section{ENSEMBLE KALMAN FILTER BASED PARTICLE FILTER}

\subsection{Particle filter}

Particle filter is also called Sequential Monte Carlo (SMC) methods. Particle filter is a set of genetic-type particle Monte Carlo methodologies to solve the filtering problem [10]. It is a probability-based filter. The key idea is to generate a given number $M$ state vectors based on the probability density function(pdf) [10].

In particle filter, the microarray data is represented in terms of the variables $y_{i}$, which are also a set of noisy observations.

A state space model is given below.

$$
\begin{aligned}
x_{i} & =f\left(x_{i-1}, t_{i-1}\right)+\nu_{i} \\
y_{i} & =h\left(x_{i}, t_{i}\right)+\mu_{i}
\end{aligned}
$$

$f($.$) is the system dynamic function. h($.$) is the system ob-$ servation function. They are nonlinear and noisy. $x_{i}$ is the state vector. $y_{i}$ is the observation vector. $\nu_{i}$ is system noise vector with covariance $Q . \mu_{i}$ is the observation noise vector with covariance $R$.

The general procedure of particle filter in GRNs analysis is given by Algorithm 1 [10].

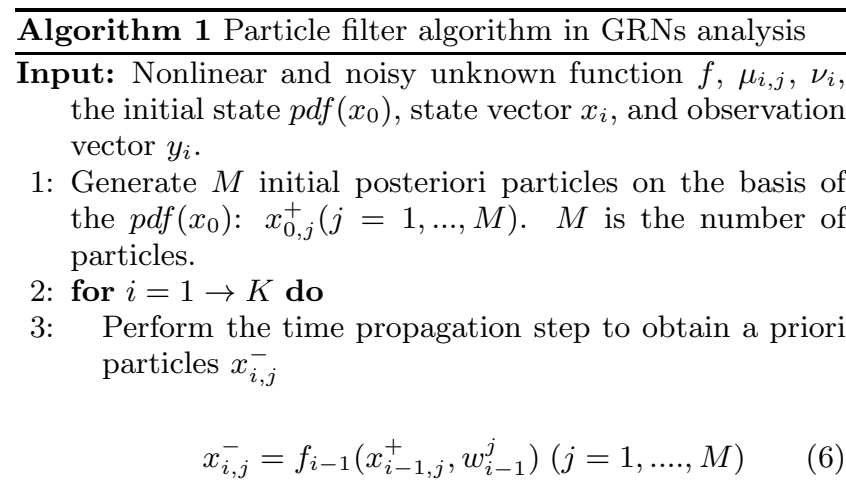

where $w_{i-1}^{j}$ is generated on the basis of pdf of $w_{k-1}$.

4: Compute the relative likelihood $q_{j}$ of each particle $x_{i, j}^{-}$ conditioned on the measurement of $y_{i}$ on the basis of nonlinear measurement equation and the pdf of the measurement noise

$$
q_{j}=\frac{1}{(2 \pi)^{L / 2}|R|} \exp \left(\frac{-\left[y^{*}-h\left(x_{i, j}^{-}\right)\right]^{T} R^{-1}\left[y^{*}-h\left(x_{i, j}^{-}\right)\right]}{2}\right)
$$

where $y^{*}$ is a specific measurement, and $L$ is the number of elements.

5: Scale the relative likelihoods

$$
q_{j}=\frac{q_{j}}{\sum_{m=1}^{M} q_{m}}
$$

6: $\quad$ Resampling step: generate posteriori particles $x_{i, j}^{+}$on the basis of the relative likelihoods $q_{i}$

7: Accept those higher importance weights particles with cumulative probabilities bigger than a given threshold.

8: Compute the mean and covariance on the basis of $x_{i, j}^{+}$ which are distributed according to the $p d f\left(x_{i} \mid y_{i}\right)$.

9: end for

10: Estimate the RMS error

$$
R M S=\left(\frac{1}{N^{2}} \sum_{i=1}^{N}\left|X_{i}^{*}-X_{i}\right|\right)^{1 / 2}
$$

where $X_{i}^{*}$ is one given measurement. 


\subsection{Ensemble Kalman filter}

The ensemble Kalman filter is one optimal estimator with error statistics predicted using ensemble integration to solve the Fokker-Planck equation [5]. Ensemble Kalman filter without a model is proposed in [6]. The proposed method can analyze time series data without access to a model. For GRNs, dynamic relations among genes are inferred from noisy training data set. Since particle filter can also analyze data without a model, modified ensemble Kalman filter in [6] is adopted. The system and state equations are the same as equations (5) in particle filter.

The modified ensemble Kalman filter is given by Algorithm $2[10,6]$.

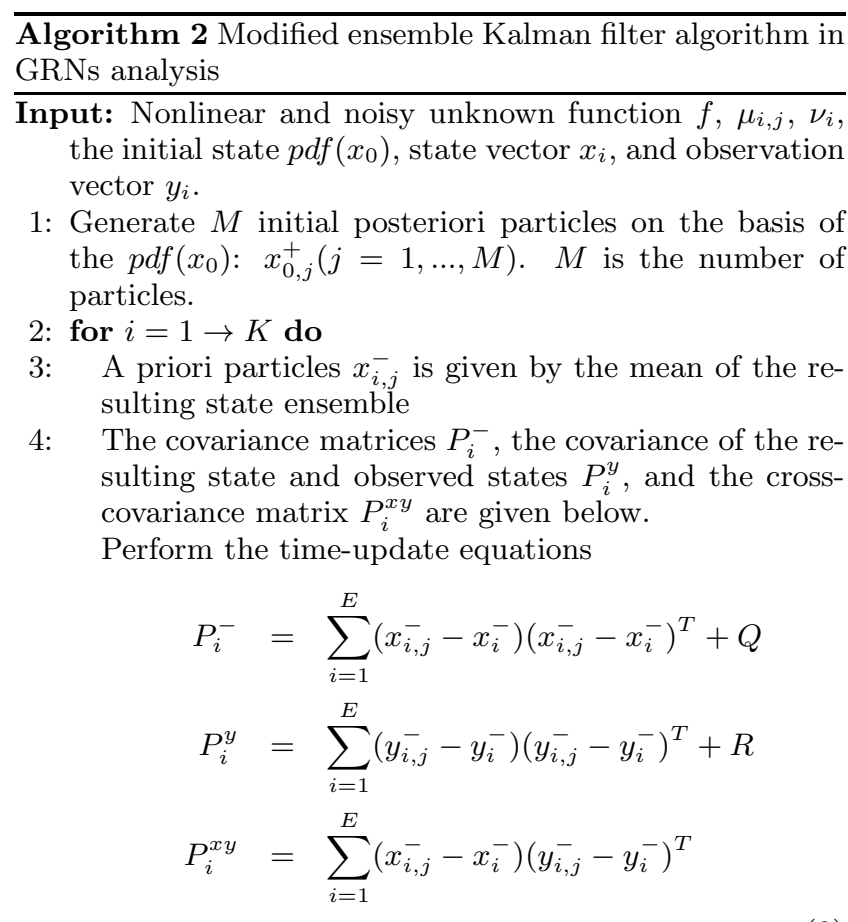

where $x_{i}^{-}$and $y_{i}^{-}$are the mean of the resulting state ensemble and the mean of the observed ensemble, respectively.

5: State and covariance are estimated with observation using following equations.

$$
\begin{aligned}
K_{i}^{-} & =P_{i}^{x y}\left(P_{i}^{y}\right)^{-1} \\
P_{i}^{+} & =P_{i}^{-}-P_{i}^{x y}\left(P_{i}^{y}\right)^{-1} P_{i}^{x y} \\
x_{i}^{+} & =x_{i}^{-}+K_{i}^{-}\left(y_{i}-y_{k}^{-}\right)
\end{aligned}
$$

6: end for

\subsection{Proposed ensemble Kalman filter based par- ticle filter}

The proposed ensemble Kalman filter based particle filter is shown in Algorithm 3. Ensemble Kalman filter is applied to predict new particles based on the state estimation in the last step of particle filter. Thus, the proposed algorithm can alleviate the sample degeneracy problem in particle filter algorithm. The proposed algorithm can also adjust gain imbalance factor by adjusting attenuation factor, which can compromise between accuracy and robustness.

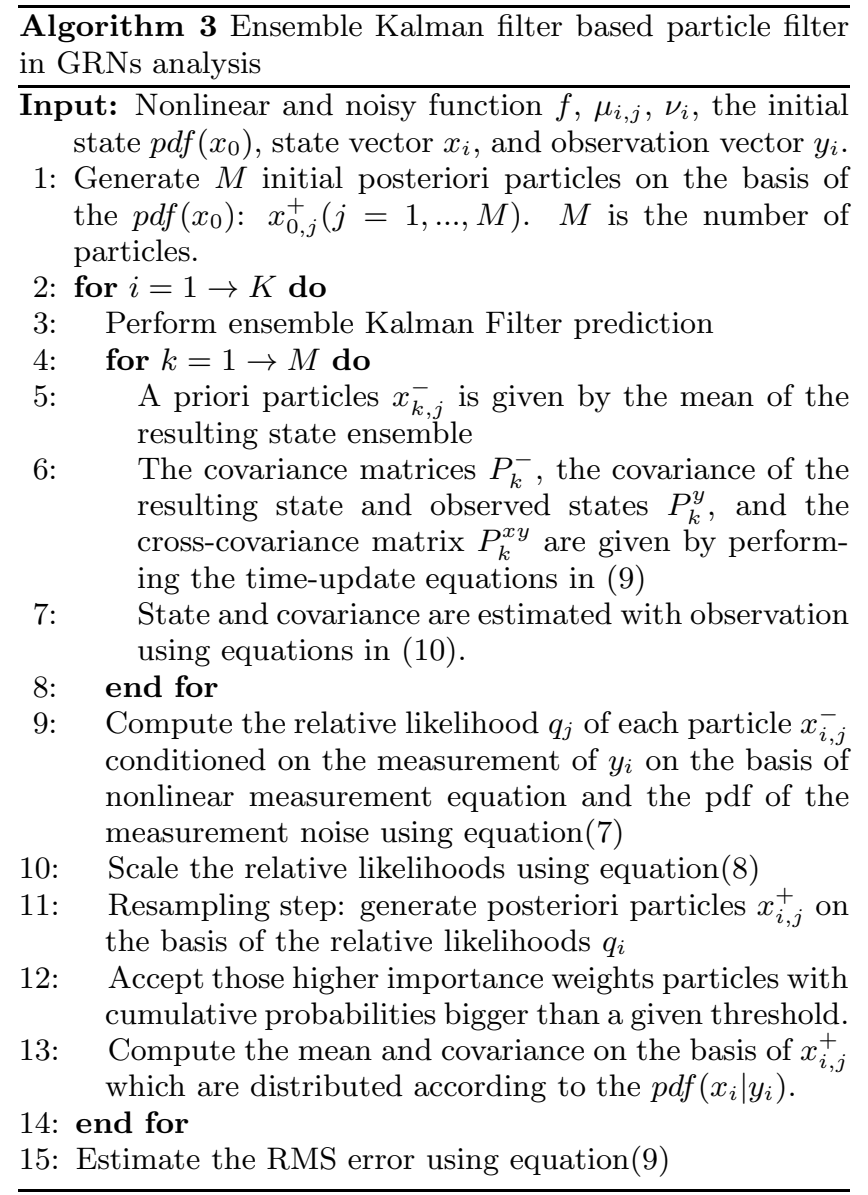

\subsection{Complexity analysis of the proposed algo- rithm}

The efficiency of the proposed algorithm is compared with the standard particle filter.

The time cost function of particle filter is $T(K, M)=$ $\sum_{i=1}^{K} \sum_{j=1}^{M} C 1=C 1 K M \in \Omega(K M)$, where $K$ is the total number of time steps. $M$ is the total number of particle in each loop. $C 1$ is the time cost for each loop of particle filter.

The time cost function of proposed algorithm is $T(K, M)=$ $\sum_{i=1}^{K} \sum_{j=1}^{M}(C 1+C 2)=(C 1+C 2) K M \in \Omega(K M)$, where $C 2$ is the time cost for each loop of the ensemble Kalman filter. Usually, if $k \ll m$, we have $T(K, M) \in \Omega(M)$. The proposed algorithm has the same level of time efficiency as the particle filter algorithm.

\section{SIMULATION RESULTS}

In order to test the proposed ensemble Kalman filter based particle filter, data from both synthetic models and real microarray experiments are used.

\subsection{Synthetic model simulation}

The GRNs model analysis using ensemble Kalman filter based particle filter is tested by synthetic data generated 
from the model in [11]. For the proposed algorithm, it is not necessary to have the exact model of $f($.$) . Noisy time$ series data is the main input for the model. The model has three inputs: gene $X 1$, gene $X 2$, and gene $X 3$. The simulation results from standard particle filter and the proposed algorithm are compared.

Fig. 1, Fig. 2, and Fig. 3 show the simulation results with noise covariance $Q=10$ and $R=0.08$. It is obvious that both standard particle filter and ensemble Kalman filter based particle filter can estimate the gene state throughout the entire simulation procedure. Ensemble Kalman filter based particle filter can estimate better results compared with the standard particle filter.

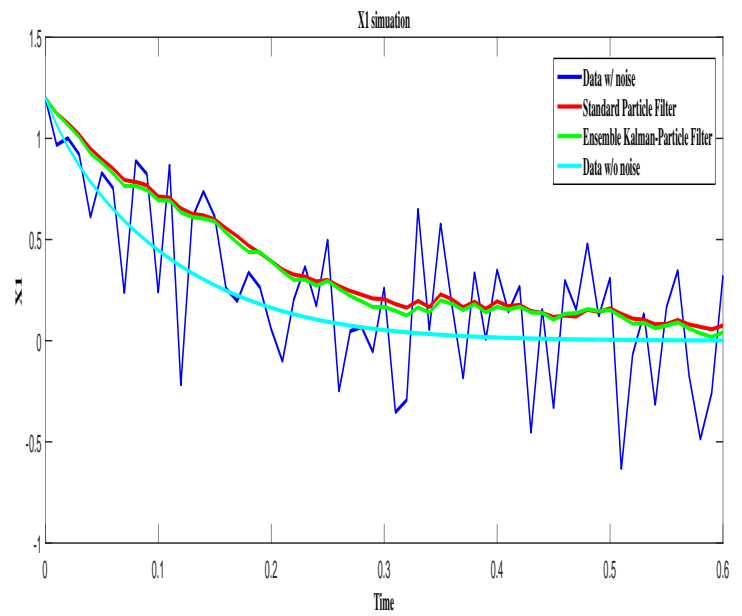

Figure 1: Synthetic simulation data for $X 1$ with Noise

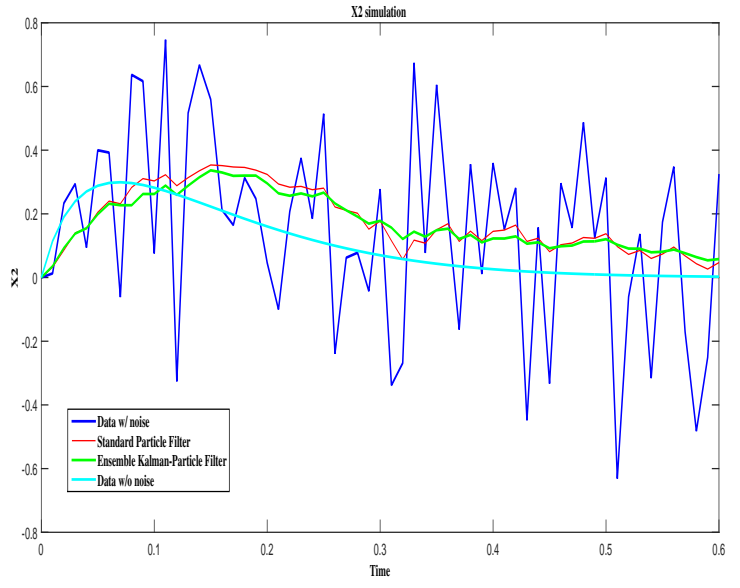

Figure 2: Synthetic simulation data for $X 2$ with Noise

Fig. 4, Fig. 5, and Fig. 6 show the estimation error based on time using the standard particle filter and the proposed algorithm . Table 1 shows the accumulative error using $R M S=\left(\frac{1}{N^{2}} \sum_{i=1}^{N}\left|X_{i}^{*}-X_{i}\right|\right)^{1 / 2}$. We can see that the proposed ensemble Kalman based particle filter can guarantee the estimation accuracy and performs better than the stan-

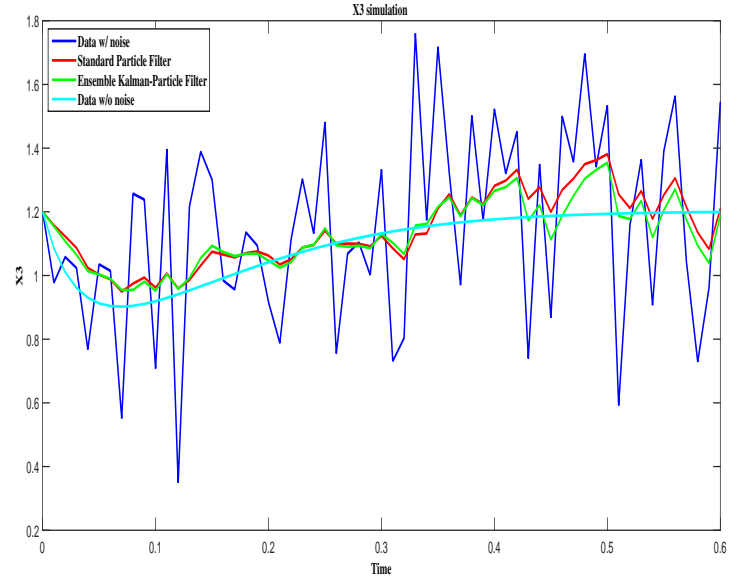

Figure 3: Synthetic simulation data for $X 3$ with Noise

dard particle filter. The reason is that the proposed method can employ current measurements. However, standard particle filter does not think over current measurements. From Fig. 4, Fig. 5, Fig. 6, and table 1, we can conclude that the proposed method is much more robust than standard particle filter and comes with satisfactory results in the whole process.

Table 1: The RMS error from standard particle filter and ensemble Kalman filter based particle filter

\begin{tabular}{|l|l|l|l|}
\hline Method & $\mathrm{X} 1$ & $\mathrm{X} 2$ & $\mathrm{X} 3$ \\
\hline $\begin{array}{l}\text { Standard particle fil- } \\
\text { ter }\end{array}$ & 18.1174 & 9.89067 & 6.86664 \\
\hline Proposed method & 16.5263 & 9.22541 & 6.61891 \\
\hline
\end{tabular}

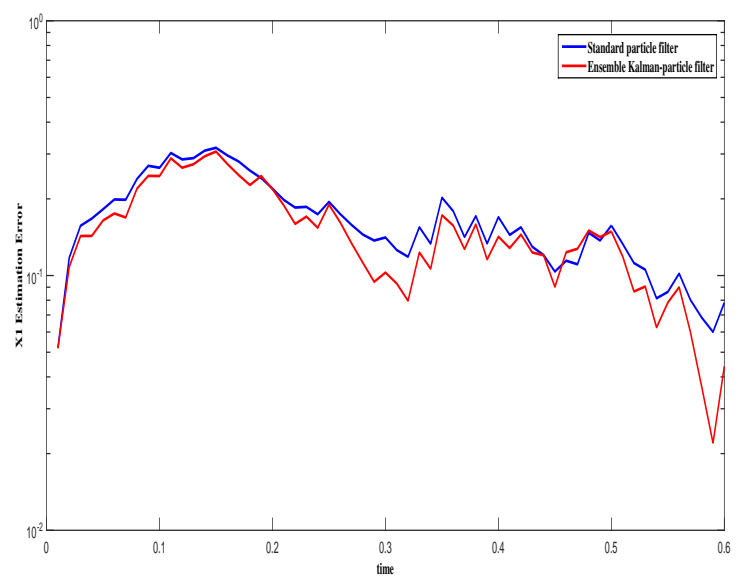

Figure 4: Estimation error of $X 1$ 




Figure 5: Estimation error of $X 2$

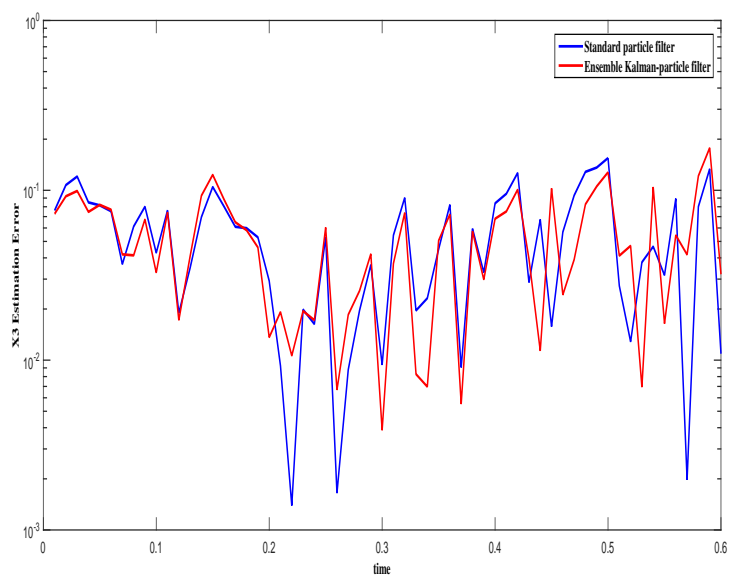

Figure 6: Estimation error of $X 3$

\subsection{Microarray data simulation}

In this part of the simulation, time-series gene-expression data corresponding to yeast protein synthesis [11] are considered. Five genes $\left(\operatorname{HAP} 1\left(X_{1}\right), \operatorname{CYB} 2\left(X_{2}\right), \operatorname{CYC} 7\left(X_{3}\right)\right.$, $\left.\operatorname{CYT} 1\left(X_{4}\right), \operatorname{COX} 5 \mathrm{~A}\left(X_{5}\right)\right)$ are selected because the relations among them have been revealed by biological experiments.

Ensemble Kalman filter based particle filter is applied to analyze the real microarray data with noise. Results are shown in Fig. 7, Fig. 8, Fig. 9, Fig. 10, and Fig. 11 for genes HAP1,CYB2,CYC7,CYT1, and COX5A. We can see that the proposed method performs well on the real nonlinear noisy microarray data.

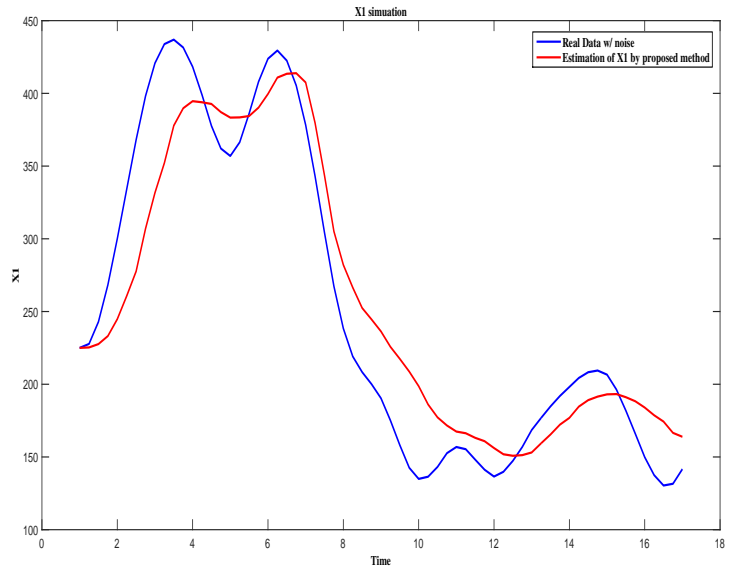

Figure 7: Microarray data simulation for HAP1

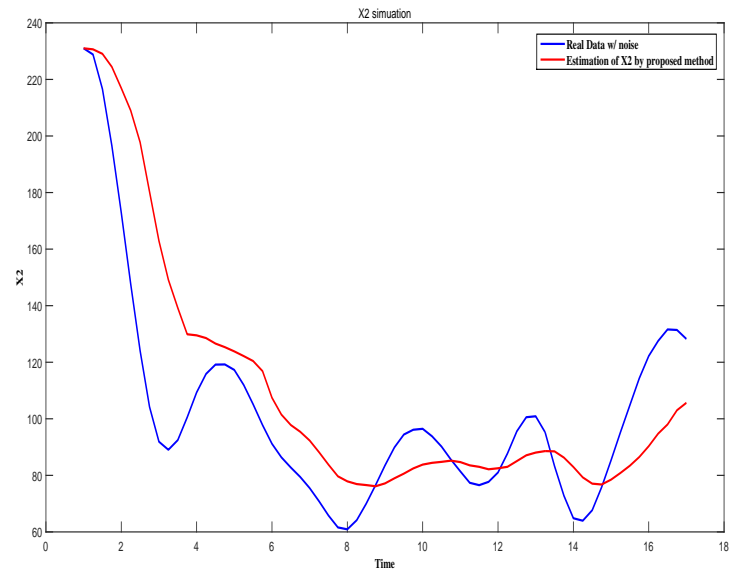

Figure 8: Microarray data simulation for CYB2

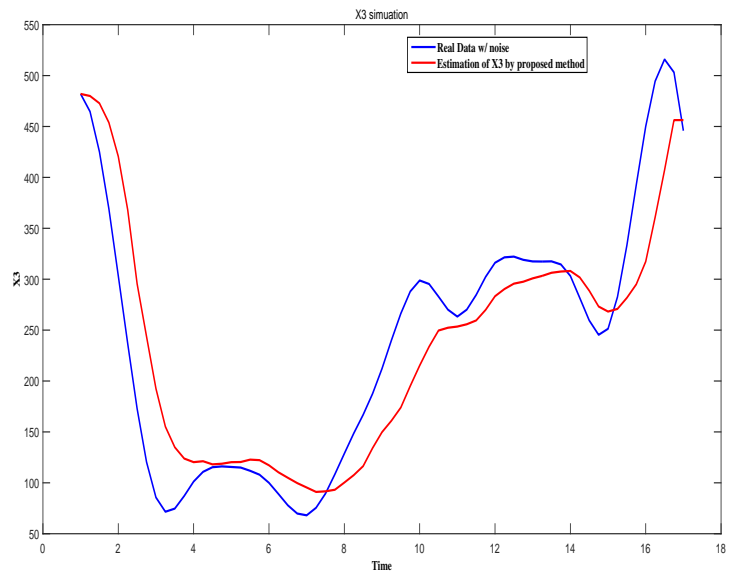

Figure 9: Microarray data simulation for CYC7 


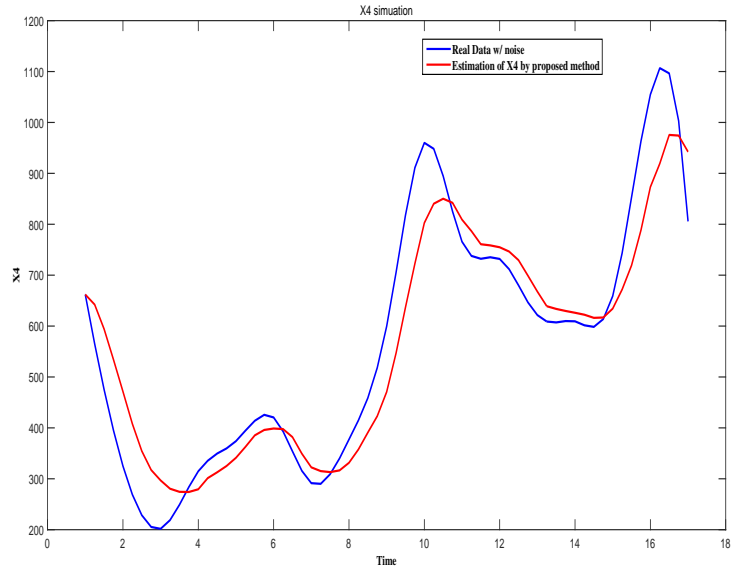

Figure 10: Microarray data simulation for CYT1

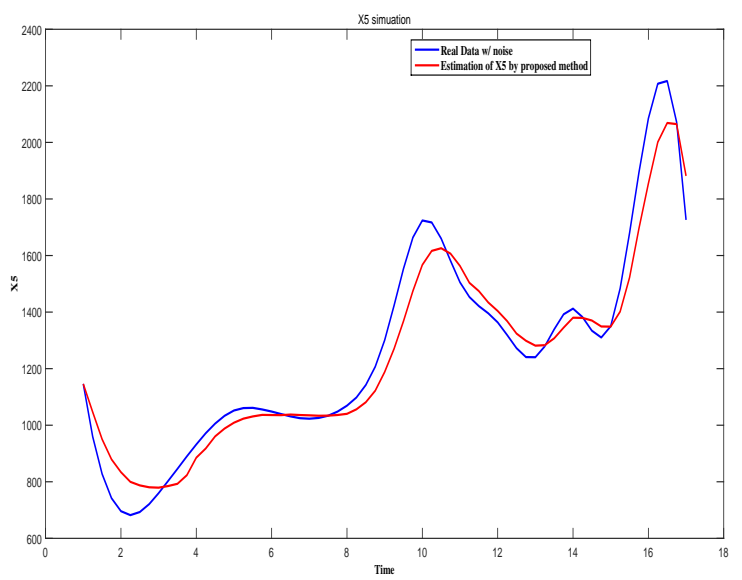

Figure 11: Microarray data simulation for COX5A

\section{CONCLUSION}

In this paper, a novel ensemble Kalman filter based particle filter is proposed. The proposed method overcomes the overfitting problem in deterministic and stochastic models. There is no sacrifice of predictive accuracy. The simulation results show that the proposed method has better accuracy, robustness, and efficiency in GRNs analysis. The proposed method takes current measures into account, which makes particle distribution more approach to the station posterior distribution. Furthermore, the proposed method can be applied to nonlinear and nonparametric forecasting models. GRNs are one of those models. Finally, both synthetic and real microarray experiment data are applied to the proposed method. Results show that the proposed algorithm is effective and practicable in GRNs analysis. The future work is to apply the proposed algorithm in gene control networks with high dimensional data.

\section{ACKNOWLEDGEMENT}

The work of Haixin Wang and Dawit Aberra was supported by NSF Grant 1435152 .

\section{REFERENCES}

[1] M. S. Dasika, A. Gupta, C. D. Maranas, and J. D. Varner. A mixed integer linear programming (milp) framework for inferring time delay in gene regulatory networks. In In Pacific Symposium on Biocomputing, pages 474-485, 2004.

[2] P. D'haeseleer and S. Fuhrman. Gene network inference using a linear, additive regulation model. 1999.

[3] P. D'haeseleer, S. Liang, and R. Somogy. Genetic network inference: from co-expression clustering to reverse engineering. Bioinformatics, 16:707-726, 2000.

[4] J. Gebert, N. Radde, and G.-W. Weber. Modeling gene regulatory networks with piecewise linear differential equations. European Journal of Operational Research, 181:1148-1165, 2007.

[5] S. Gillijns, K. Universiteit, and etc. What is the ensemble kalman filter and how well does it work? IEEE 2006 American Control Conference, 2006.

[6] F. Hamilton, T. Berry, and T. Sauer. Ensemble kalman filtering without a model. Physical Review X, 6, 2016.

[7] S. Kimura, K. Ide, A. Kashihara, M. Kano, M. Hatakeyama, R. Masui, N. Nakagawa, S. Yokoyama, S. Kuramitsu, and A. Konagaya. Inference of s-system models of genetic networks from noisy time-series data. Chem-Bio Informatics Journal, 4(1):1-14, 2004.

[8] Z. Kutalik, W. Tucker, and V. Moulton. S-system parameter estimation for noisy metabolic profiles using newton-flow analysis.

[9] L. Qian, H. Wang, and E. Dougherty. Inference of noisy nonlinear differential equation models for gene regulatory networks using genetic programming and kalman filtering. IEEE Transactions on Signal Processing, 56(8):3327-3339, 2008.

[10] D. Simon. Optimal State Estimation Kalman, $H_{\mathrm{inf}}$ and Nonlinear Approaches. Wiley, 2006.

[11] H. Wang and D. Aberra. Noise analysis of gene regulatory networks using particle filter. International Journal of Clinical Biostatistics and Biometrics, pages 1-10, 2015.

[12] H. Wang, L. Qian, and E. Dougherty. Modeling genetic regulatory networks by sigmoidal functions: A joint genetic algorithm and kalman filtering approach. icnc, 2:324-328, 2007.

[13] H. Wang, L. Qian, and E. Dougherty. Inference of gene regulatory networks using s-system. IET System Biology, 4(2):145-156, 2010. 